\title{
Teste de Usabilidade de Jogos para Ensino-Aprendizado de Lógica Matemática
}

\author{
Bruna de Abreu Dias \\ Universidade Federal do Pampa \\ Alegrete, Rio Grande do Sul, BR \\ brunadias.aluno@unipampa.edu.br
}

\author{
Alice Fonseca Finger \\ Universidade Federal do Pampa \\ Alegrete, Rio Grande do Sul, BR \\ alicefinger@unipampa.edu.br
}

\begin{abstract}
According to the Brazilian Computer Society, the number of new students in the computer area courses increases in the last years. On the order hand, the Brazilian numbers and other countries point to high dropout rates in these courses. The goal of managers and teachers is to know the student's reasons for dropout and to reduce retention rates. The teachers could use different teaching strategies to motivate students and learning assistance, such as games. The goal of this work is to evaluate the usability of Andor: Learn Logic and Trybit Logic applications for teaching Mathematical Logic. The test was applied with different users and questions about the using applications were responded to. The results show that the Trybit Logic application presents better quality and easy to use. The users consider it to be an easy to use, and intuitive game that, which can be useful in teaching and learning Mathematical Logic because use logical operators in a fun way.
\end{abstract}

\section{KEYWORDS}

Lógica Matemática, Jogos para Ensino-Aprendizagem, Jogos de Lógica Matemática, Teste de Usabilidade

\section{INTRODUÇÃO}

Os cursos de graduação na área da Computação despertam o interesse de muitos jovens que estão escolhendo uma área para seguir. Entretanto, ao encontrar os desafios que as disciplinas com viés matemático e lógico apresentam, os discentes muitas vezes tendem a evadir do curso escolhido ou retém-se nas disciplinas pois consideram um empecilho difícil de ser ultrapassado.

Dados quantitativos apresentando a série histórica dos cursos de Computação no Brasil são disponibilizados pela Sociedade Brasileira de Computação [1]. Na última estatística encontrada foi possível perceber um aumento no número de cursos de graduação criados nessa área. Entretanto, chamam a atenção os dados referentes ao número de ingressantes e concluintes desses cursos. Se olharmos os dados relativos aos últimos anos apresentados, 2017 e 2018, percebese um aumento no número de ingressantes, porém, o número de concluintes é sempre muito baixo, mesmo naqueles cursos onde houve aumento de um ano para outro.

No que diz respeito às instituições públicas, por exemplo, compreender esse fenômeno pode contribuir significativamente na diminuição do desperdício de recursos. Segundo [2], muitas vezes se descobre tarde a intenção do discente de evadir, acontecendo somente após a saída do aluno do curso ou quando o discente já está com a certeza de deixar o curso ou a instituição. Assim, é imprescindível que os gestores atuem na função de conhecer as causas da evasão discente para que possam tomar medidas que tentem diminuir esses altos índices.

O problema deve ser tratado em diferentes níveis dentro de uma instituição de ensino. Os professores também devem ser capazes de identificar causas e propor melhorias, qualificando suas metodologias de ensino bem como estreitando laços com os alunos, a fim de precocemente identificar problemas que possam causar evasão ou retenção em disciplinas.

É conhecido que em cursos da área de Computação a taxa de retenção e evasão em disciplinas com viés teórico e matemático é maior. Ao encontro do que foi relatado anteriormente, é fundamental e necessário que os docentes consigam utilizar estratégias de ensino mais atrativas para que os discentes possam enxergar essas disciplinas como algo que não pareça tão desafiador. O uso de jogos para apoiar o ensino e aprendizagem pode ser uma boa estratégia e tem sido muito utilizada em disciplinas como programação, por exemplo [3].

No que diz respeito à disciplina de Lógica Matemática (LM), foram encontrados poucos relatos na literatura de softwares que auxiliam no ensino do conteúdo. Porém, uma busca por aplicativos na Google Play Store retornou diversas aplicações que abordam tópicos de LM. A partir de uma revisão sistemática acerca desses resultados, foi possível identificar algumas categorias para os aplicativos [4].

A realização de um teste de usabilidade é um processo com atividades definidas que objetivam coletar dados da interação do usuário com o produto. [5] Com base nos resultados obtidos, podese detectar problemas na usabilidade. [6]. Ainda, em relação aos aplicativos que serão utilizados no presente trabalho, acrescenta-se que os mesmos têm como objetivo a possibilidade de saber qual apresenta uma melhor usabilidade, a fim de torná-lo um aliado no ensino e aprendizado na disciplina de Lógica Matemática.

Na seção 2 são apresentados os principais trabalhos relacionados com o tema do presente artigo. Já na seção 3 são apresentadas as etapas utilizadas durante o desenvolvimento do teste de usabilidade. Na sequência, a seção 4 traz os resultados obtidos a partir da aplicação do teste, juntamente com as discussões acerca desses resultados, descritas na seção 5 . Por fim, na seção 6 são apresentadas as considerações finais, seguidas das principais referências.

\section{TRABALHOS RELACIONADOS}

Esta seção tem como objetivo apresentar os trabalhos encontrados na literatura com foco no ensino de lógica matemática, teste de usabilidade, bem como o uso de jogos para o auxílio do ensinoaprendizado.

No trabalho desenvolvido por [7], os autores identificaram que materiais complexos e altamente abstratos para a aprendizagem 
de matemática são um motivo para a alta taxa de abandono nos primeiros semestres de cursos da área de Computação. Assim, no intuito de apoiar a compreensão e prática dos principais conceitos matemáticos, desenvolveram um ambiente de aprendizagem baseado em jogo multijogador que oferece suporte à competição, colaboração e cooperação. O protótipo foi testado com dez participantes, divididos em cinco grupos. Após o tempo de uso, os estudantes foram questionados sobre o que gostavam e não gostavam no jogo e o que poderia ser melhorado. Como resultado, mesmo com o relato de alguns problemas de usabilidade, o jogo foi percebido pelos usuários como motivador e divertido.

O objetivo principal do trabalho de [8] foi construir e validar uma pesquisa de avaliação de usabilidade para ambientes de aprendizagem móvel. Foi desenvolvido um questionário com 42 questões em escala Likert, dividido em 8 atributos de usabilidade: eficácia, eficiência, oportunidade, satisfação, capacidade de aprendizagem, memorização, erro e carga cognitiva. A partir de uma verificação empírica, obtiveram um consenso de quatorze especialistas quanto aos itens do questionário. Os resultados indicam que mais de $88 \%$ dos especialistas consentiram em todos os itens de usabilidade representados no questionário. Os autores afirmam que a pesquisa pode ajudar a melhorar a satisfação do usuário e reduzir os custos de treinamento, o que atrai muitos pesquisadores, designers de interface e gerentes de projeto para empregar a avaliação de usabilidade ao projetar as interfaces para aplicativos de aprendizagem.

Os autores de [9] realizaram experimentos com jogo educacional para o aprendizado de área de medição de software da Engenharia de Software. Analisando resultados a partir de testes estatísticos, concluíram que do ponto de vista qualitativo, os dados demonstraram que mais participantes indicaram ter gostado de utilizar o jogo do que o contrário. Antes da realização dos experimentos eles acreditavam que a utilização do jogo educacional pudesse causar um efeito positivo significativo em termos de aprendizagem em acadêmicos de graduação na área de Computação. Porém, após o experimento, ficou evidente que o efeito de aprendizagem nos níveis de conhecimento, compreensão e aplicação do grupo experimental não são significativamente superiores aos do grupo de controle.

Considerando a falta de motivação dos alunos nas aulas de matemática, o trabalho de [10] objetivou mobilizar conhecimentos matemáticos através da construção de jogos digitais pelos alunos da educação básica. Como resultado salienta-se o desenvolvimento da autonomia do aluno na aprendizagem, além disso, as autoras destacam que jogos digitais são ferramentas atraentes para os alunos e fornecem subsídios para construir significados na aprendizagem.

Em [6] encontra-se o relato de um teste de usabilidade realizado em uma aplicação móvel educacional desenvolvida para Android, Education Hub. A aplicação foi desenvolvida para professores, alunos e instituições de ensino para promover o ensinoaprendizagem via web. Os autores descrevem a execução, etapas e questões que envolveram o desenvolvimento do teste antes de seu lançamento no mercado. O objetivo do trabalho foi o incentivo às empresas para realização de mais testes de usabilidade. Dos resultados obtidos, destaca-se que os usuários tiveram dificuldade no reconhecimento de ícones mas a maioria concordou que o aplicativo seria útil no aprendizado.

É possível observar que entre os trabalhos citados não foram encontradas menções ao ensino de Lógica Matemática através de jogos para smartphones. Por isso, neste artigo, objetivamos apresentar um teste de usabilidade, o qual ajudará a definir qual jogo pode servir como auxílio no entendimento da disciplina.

\section{METODOLOGIA}

A presente seção tem como objetivo descrever a metodologia utilizada no trabalho. A partir de uma investigação sobre aplicativos para dispositivos móveis (com o Sistema Operacional Android) que auxiliem no ensino-aprendizagem de Lógica Matemática [4], o resultado mostrou que, dos quatro jogos avaliados, Andor: Learn Logic e Trybit Logic apresentam características mais relevantes, tais como: tutoriais que auxiliam significativamente no entendimento do jogo e grande quantidade de níveis. Assim, esses dois aplicativos foram escolhidos para o teste de usabilidade, no intuito de avaliar problemas e aspectos para melhoria, bem como apontar qual deles teria melhor usabilidade frente aos usuários para possível auxílio na disciplina de Lógica Matemática. As subseções abaixo descrevem os jogos utilizados e o processo envolvendo o teste de usabilidade.

Foram criados dois cenários, um para cada jogo apresentado pois se tratavam de aplicações com objetivos e conteúdos distintos. Os cenários consistem em passos que o usuário deve seguir durante o teste para posterior análise do jogo. Após os usuários finalizarem cada cenário, foi enviado um formulário com questões criadas pelas autoras e outras questões que são encontradas em [6].

Tendo em vista o uso da Escala Likert [11] como resposta no formulário, ao fim do teste, as respostas foram analisadas e a mediana calculada para realização do comparativo entre os jogos. Detalhes do teste de usabilidade são encontrados na seção 3.2.

\subsection{Andor: Learn Logic e Trybit Logic}

O jogo Andor: Learn Logic, disponível para download na Google Play Store [12], possui tamanho de $2,6 \mathrm{MB}$ e tem como idioma principal o inglês, tendo disponibilidade de uso sem internet e sua última atualização sendo em 2018. O jogo contém 31 níveis de tutorial onde são apresentados seus 6 operadores lógicos e explicado o uso dos parênteses conforme o avanço. Ele consiste na reorganização de premissas de maneira que fiquem verdadeiras, conforme Fig. 1. Salienta-se que as figuras apresentadas na presente subseção são capturas de tela dos jogos com ilustrações para um melhor entendimento do que está sendo destacado. A partir desta seção o jogo "Andor: Learn Logic" será chamado somente de "Andor" para facilitar leitura.

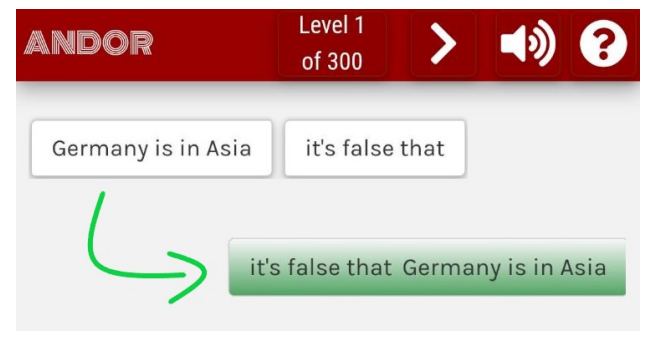

Figure 1: Andor: ilustração da frase desorganizada e resolvida 
Andor contém um total de 300 níveis e, após o término do tutorial, é possível que o jogador utilize o "Symbolic Mode", Fig. 2, onde são apresentadas as premissas como true ou false e os operadores com suas respectivas representações simbólicas, os quais anteriormente eram encontradas apenas com sua escrita em inglês.

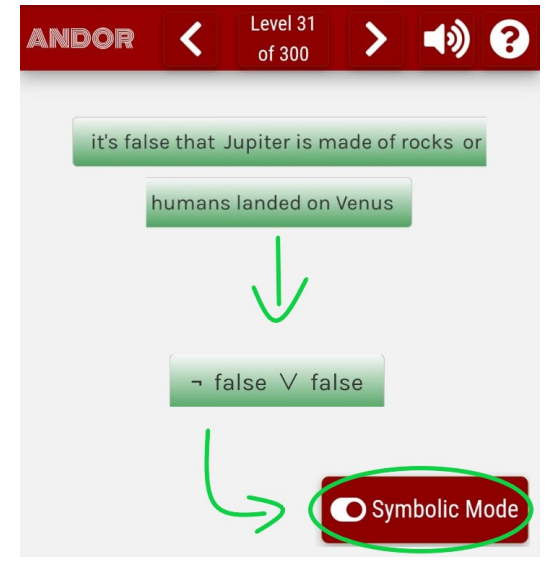

Figure 2: Andor: ilustração da mudança que ocorre nos operadores e premissas no uso do Symbolic Mode

O jogo Trybit Logic, disponível para download também na Google Play Store [13], possui como idioma principal o inglês, com tamanho de $45 \mathrm{MB}$, tendo sua última atualização em 2019 e possibilitando uso sem internet. Nele tem-se como foco o ensino de 4 operadores lógicos através da derrota de bugs que pretendem destruir o mascote do jogo, o robô BITROBO. Em seu tutorial, o jogo apresenta o funcionamento dos painéis de bit dos bugs e dos operadores lógicos encontrados. Existem ainda dois modos de jogo, o "Defense Mode" e o "Puzzle Mode", conforme Fig. 3, que diferem na forma de derrota do bug, pois no primeiro modo é necessário derrotá-lo conforme o avanço dos seus painéis de bit, arrastando os painéis dos operadores pela tela. Já no segundo modo, o jogo é estático e contém somente restrições de movimento dos painéis de bit dos bugs e dos operadores.

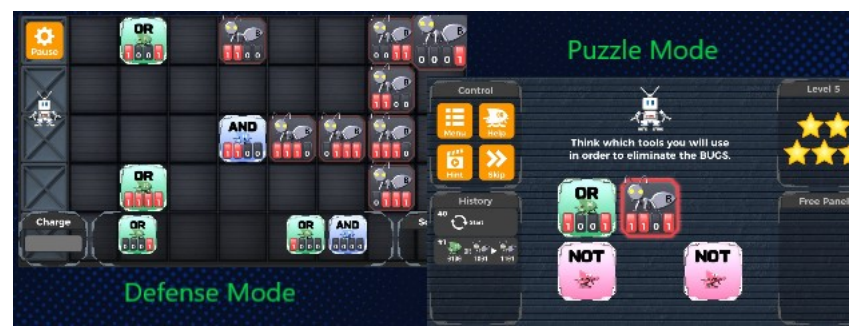

Figure 3: Trybit Logic: ilustração dos modos Defense Mode e Puzzle Mode

\subsection{Teste de Usabilidade}

Para a execução do teste de usabilidade, primeiramente, analisouse cada jogo para construção dos cenários, assim seria possível abranger as funções e particularidades de cada aplicação. Com base no passo a passo definido, foram criadas questões específicas, disponíveis na Tab. 1 e na Tab. 2, uma vez que os jogos apresentam diferentes formas de manipulação. As tabelas que contêm as questões utilizadas tem em sua primeira coluna o código correspondente a questão e na segunda coluna a sua descrição.

\begin{tabular}{|l|l|}
\hline \multicolumn{2}{|l|}{ Seção A: Andor } \\
\hline A.1 & Os sons dificultaram a concentração durante o jogo \\
\hline A.2 & Foi fácil encontrar a "Ajuda" \\
\hline A.3 & Foi fácil desabilitar o som \\
\hline A.4 & Foi fácil navegar entre os níveis \\
\hline A.5 & Foi fácil sair do jogo \\
\hline \\
Table 1: Questões específicas para o jogo Andor
\end{tabular}

\begin{tabular}{|l|l|}
\hline \multicolumn{2}{|l|}{ Seção A: Trybit Logic } \\
\hline Puzzle Mode \\
\hline A.1 & Foi fácil iniciar o jogo \\
\hline A.2 & Os sons dificultaram a concentração durante o jogo \\
\hline A.3 & $\begin{array}{l}\text { As animações dificultaram a concentração durante } \\
\text { o jogo }\end{array}$ \\
\hline A.4 & Foi fácil encontrar a "Ajuda" por operador \\
\hline A.5 & Foi fácil desabilitar o som \\
\hline A.6 & $\begin{array}{l}\text { Foi fácil, ao finalizar um nível, reiniciá-lo sem ir ao } \\
\text { menu inicial }\end{array}$ \\
\hline A.7 & Foi fácil retomar o início do nível sem sair da tela atual \\
\hline A.8 & Foi fácil sair do jogo \\
\hline Defense Mode \\
\hline A.9 & Foi fácil reiniciar o jogo \\
\hline A.10 & $\begin{array}{l}\text { Foi fácil encontrar "Ajuda" para os operadores } \\
\text { disponíveis }\end{array}$ \\
\hline
\end{tabular}

Table 2: Questões específicas para o jogo Trybit Logic

Também foram utilizadas questões mencionadas em [6], disponíveis na Tab. 3 e na Tab. 4.

\begin{tabular}{|l|l|}
\hline \multicolumn{2}{|l|}{ Seção B: Facilidade de Uso } \\
\hline B.1 & Foi fácil aprender a utilizar o aplicativo \\
\hline B.2 & $\begin{array}{l}\text { Eu conseguia entender o que acontecia durante o } \\
\text { uso do aplicativo }\end{array}$ \\
\hline B.3 & $\begin{array}{l}\text { Foi fácil ganhar habilidade de uso durante a } \\
\text { execução das atividades no aplicativo }\end{array}$ \\
\hline B.4 & É fácil lembrar como utilizar o aplicativo \\
\hline B.5 & Considero o aplicativo fácil de utilizar \\
\hline Seção C: Utilidade do Aplicativo \\
\hline C.1 & $\begin{array}{l}\text { Considero o aplicativo útil para melhorar } \\
\text { meu aprendizado }\end{array}$ \\
\hline C.2 & $\begin{array}{l}\text { Considero que o aplicativo melhoraria minha } \\
\text { produtividade para realização das atividades e } \\
\text { aprendizado }\end{array}$ \\
\hline C.3 & $\begin{array}{l}\text { Considero que o aplicativo facilitaria a realização } \\
\text { das minhas atividades }\end{array}$ \\
\hline
\end{tabular}

Table 3: Questões baseadas em [6]

Após criação dos cenários, foram definidos os usuários do teste por aqueles discentes que já cursaram ou estavam cursando a disciplina de LM, pois teriam conhecimento do conteúdo a ser abordado. Foi criado um formulário para coletar quais seriam os interessados em participar do teste de usabilidade. Dos pré-requisitos, o discente 


\begin{tabular}{|l|l|}
\hline \multicolumn{2}{|c|}{ Seção D: Interface do Aplicativo } \\
\hline D.1 & Considero as cores e botões do aplicativo agradáveis \\
\hline D.2 & $\begin{array}{l}\text { Consigo visualizar bem todos os botões e informações } \\
\text { dentro do aplicativo }\end{array}$ \\
\hline D.3 & $\begin{array}{l}\text { Entendo com facilidade as palavras, nomenclaturas } \\
\text { e ícones do aplicativo }\end{array}$ \\
\hline D.4 & $\begin{array}{l}\text { As imagens e ícones no aplicativo são de fácil } \\
\text { reconhecimento }\end{array}$ \\
\hline D.5 & $\begin{array}{l}\text { Consigo visualizar todas as funcionalidades do } \\
\text { aplicativo }\end{array}$ \\
\hline D.6 & Consigo navegar bem por todas as telas do aplicativo \\
\hline Seção E: Ensino em Sala de Aula \\
\hline E.1 & $\begin{array}{l}\text { Após me acostumar com o aplicativo julgo que o } \\
\text { mesmo facilita o ensino e aprendizagem: }\end{array}$ \\
\hline E.2 & $\begin{array}{l}\text { Com o aplicativo considero que as aulas ficariam mais } \\
\text { interessantes }\end{array}$ \\
\hline E.3 & $\begin{array}{l}\text { Com o aplicativo eu conseguiria explorar bem os } \\
\text { materiais disponíveis }\end{array}$ \\
\hline E.4 & $\begin{array}{l}\text { Com o aplicativo eu teria maior domínio sobre as } \\
\text { atividades em sala }\end{array}$ \\
\hline E.5 & Com o aplicativo o nível de aprendizagem aumentaria \\
\hline
\end{tabular}

Table 4: Questões baseadas em [6]

deveria possuir acesso a um smartphone Android que possibilitasse a instalação dos dois jogos, com tamanhos de $45 \mathrm{MB}$ e 2,6MB. Eles também deveriam compreender a leitura em inglês, pois é o idioma de ambos jogos, além de assinar um Termo de Consentimento para que fosse possível realizar a gravação do teste, já que ele foi realizado de maneira remota, através da plataforma Google Meet.

O teste de usabilidade ocorreu no dia 2 de novembro de 2020 , com duração de aproximadamente 50 minutos, e contou com 6 participantes, respeitando o mínimo recomendado por [14], os quais são discentes dos cursos de Engenharia de Software e Ciência da Computação. Um e-mail foi enviado aos participantes solicitando a instalação dos jogos, bem como orientando para que eles não fossem manipulados antes do teste para evitar que os resultados fossem afetados. Salienta-se que no início do teste foram realizadas breves explicações sobre os operadores lógicos e sua semântica para que os usuários pudessem relembrar sobre o conteúdo que seria tratado. Também deseja-se destacar que todos os 6 usuários presentes testaram ambas as aplicações utilizando os mesmo cenários criados.

\section{RESULTADOS}

Esta seção objetiva apresentar os dados coletados durante o teste de usabilidade, apresentando-os através de gráficos e tabelas com a intenção de compara-los. Em seguida, será realizada uma análise sobre esses comparativos juntamente com apontamentos sobre os jogos a fim de discutir os resultados obtidos.

Para que as notas dadas pelos usuários pudessem ser melhor estudadas e exibidas, foi realizado o cálculo da mediana entre os valores obtidos na Escala Likert [11]. A mediana consiste em buscar, entre um conjunto de valores, o valor que está no meio. A partir da mediana encontrada em cada seção de questões, foram construídos gráficos para comparação dos dados referentes ao teste de usabilidade.
Na seção A, existem questões que se assemelham, por exemplo, as questões A.5 de Andor e A.8 de Trybit Logic que mencionam a facilidade do usuário em sair do jogo. Desse modo, essas questões foram especializadas e gerou-se a Fig. 4 e a Fig. 5, as quais apresentam no seu eixo $\mathrm{x}$ os jogos aqui discutidos.

A Fig. 4 apresenta em azul a questão "Foi fácil sair do jogo" e em vermelho "Facilidade em encontrar ajuda", que abrange as questões de código A.2 da Tab. 1 e de códigos A.4 e A.10 da Tab. 2. Pode-se observar que, segundo os usuários, em ambas questões o jogo Andor fornece uma melhor opção de saída e ajuda aos seus jogadores.

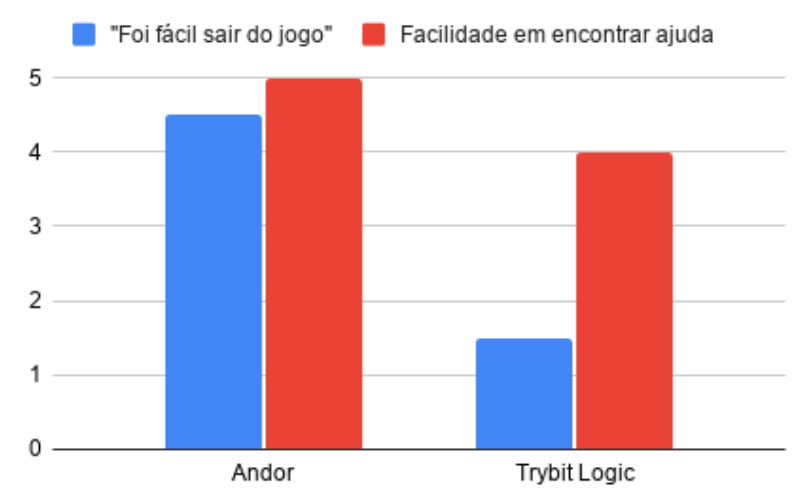

Figure 4: Gráfico comparativo de questões semelhantes da seção A de Andor e Trybit Logic

Na Fig. 5 encontra-se a questão "Dificuldade com os sons do jogo" em azul e em vermelho "Facilidade em desabilitar o som". Quanto à questão "Facilidade em desabilitar o som" ambos os jogos foram bem avaliados. Porém, nas duas questões, o jogo Trybit Logic obteve uma mediana mais alta. O que não significa algo positivo, mas sim que os sons do jogo Trybit trouxeram um maior desconforto aos usuários.

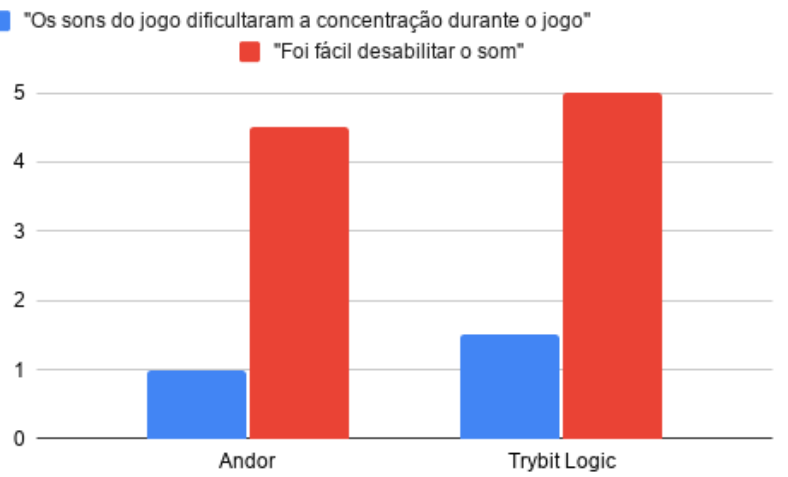

Figure 5: Gráfico comparativo de questões semelhantes da seção A de Andor e Trybit Logic

Existem questões da seção A de Andor e Trybit que não possuem semelhanças entre si e seus resultados estão na Tab. 5, na qual a 
segunda coluna representa o código de cada questão e a terceira o valor encontrado para a mediana das respostas.

\begin{tabular}{|c|c|c|}
\hline Seção A & Código & Mediana \\
\hline Andor & A.4 & 3 \\
\hline \multirow{4}{*}{ Trybit Logic } & A.1 & 5 \\
\cline { 2 - 3 } & A.3 & 1,5 \\
\cline { 2 - 3 } & A.6 & 5 \\
\cline { 2 - 3 } & A.7 & 5 \\
\cline { 2 - 3 } & A.9 & 4,5 \\
\hline
\end{tabular}

Table 5: Andor e Trybit Logic - Seção de Questões A

Pode-se observar na Tab. 5 que a única questão do jogo Andor, a qual trata sobre navegabilidade, obteve mediana igual a 3. Já em relação ao jogo Trybit Logic, quanto ao Puzzle Mode, estão as questões que tratam sobre: facilidade de iniciar o jogo; dificuldade de concentração com as animações; facilidade de reiniciar um nível sem ir ao menu e facilidade de retomar o início do nível sem mudar de tela, das quais a única que obteve uma mediana baixa foi a questão sobre dificuldade de concentração. Por fim, em relação ao Defense Mode, tem-se a questão que trata sobre a facilidade de reiniciar o jogo, a qual resultou em uma mediana 4,5.

A Fig. 6 apresenta de forma comparativa o resultado da mediana obtida em cada categoria, de B a E. O jogo Andor está representado pela cor azul e o jogo Trybit Logic pela cor vermelha.

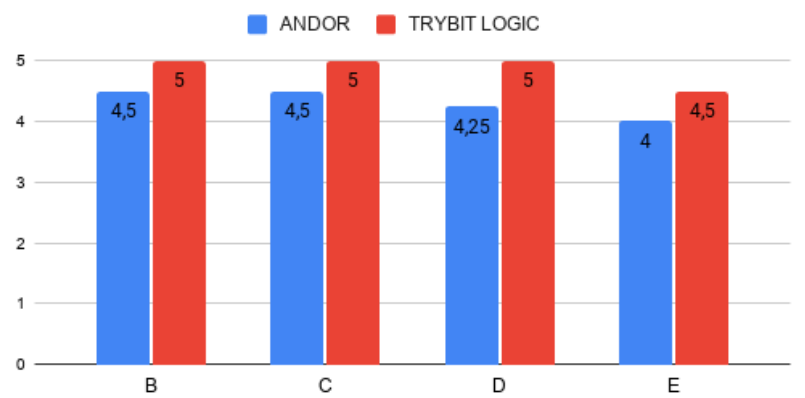

Figure 6: Gráfico comparativo de questões das Seções B até E de Andor e Trybit Logic

Nas categorias Facilidade de Uso (B), Utilidade do Aplicativo (C), Interface do Aplicativo (D) e Ensino em Sala de Aula (E), observa-se que o aplicativo Trybit Logic obteve um melhor desempenho. Já na categoria E, correspondente ao ensino, ambas aplicações obtiveram uma nota mais baixa comparado às demais categorias.

As diferentes questões referentes a seção A são difíceis de serem mensuradas, pois existem em quantidades e conteúdos distintos. Ainda assim, pode-se notar que a única questão de Andor retornou uma mediana 3 e a maioria das questões de Trybit Logic retornaram mediana 5. Na categoria A, dos comparativos observados na Fig. 4 e na Fig. 5, em 2 questões o jogo Andor foi melhor avaliado, já nas outras questões, o jogo Trybit Logic obteve uma mediana mais alta. Das categorias de B a E, é visível a diferença entre os jogos e, apesar de ambos terem obtido uma mediana mais baixa na categoria $\mathrm{E}$,
Ensino em Sala de Aula, o jogo Trybit Logic se apresenta como o jogo que obteve uma melhor apresentação para os usuários. Sendo assim, o jogo Trybit Logic foi o melhor avaliado no teste.

\section{DISCUSSÕES}

Pretende-se nesta seção discutir as medianas obtidas na seção anterior, apresentando imagens referentes aos jogos para que seja possível entender as notas atribuídas pelos usuários durante o teste de usabilidade. Assim como na seção 3, foram construídas ilustrações sobre os jogos para melhor explicar o que ocorre no seu funcionamento.

Primeiramente, é necessário enfatizar que para o artigo [4], não foram realizados nenhum tipo de teste com usuários. A análise encontrada no trabalho anterior foi construída com base em informações sobre as aplicações encontradas na página de download de cada uma (disponibilizadas pela Google Play Store). O contato ocorrido somente teve como finalidade observar se o jogo seria de fácil manipulação e até mesmo verificar características que se destacassem, como por exemplo, tutorial para auxiliar o usuário no entendimento do jogo (onde foi observado que haviam aplicações que não o continham). O teste de usabilidade realizado busca, em um grupo de usuários, destacar possíveis problemas nos jogos analisados, bem como indicar se são úteis no ensino da disciplina de Lógica Matemática.

Das medianas obtidas na primeira parte da seção A, que estão presentes na Fig. 4, nota-se que o jogo Andor obteve uma melhor avaliação quanto a sua questão A.2 referente a "Facilidade de Encontrar Ajuda". Isso ocorreu principalmente pelo fato de que os 31 níveis de tutorial do jogo apresentam reforços quanto às explicações sobre os operadores lógicos e também devido à sua interface ser simples, não contendo outras telas e sendo de fácil localização, como mostra o item 1 da Fig. 7. A ajuda para o jogo Trybit Logic não é difícil de ser encontrada, porém, ela somente está disponível enquanto um nível está sendo jogado. Além disso, o jogo apresenta algumas instruções cada vez que um nível é iniciado ou reiniciado.

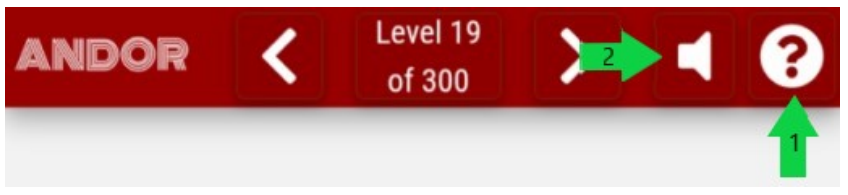

Figure 7: Jogo Andor: indicação de "som" e "ajuda"

Ao início do teste de usabilidade, foi solicitado aos usuários que não utilizassem botões do smartphone, acredita-se que isso foi descumprido, pois ao observar a Fig. 4, nota-se que foram atribuídas notas em relação a questão "Foi fácil sair do jogo", porém ambos os jogos não possibilitam a saída através da própria aplicação.

Tratando-se da Fig. 5, observa-se que Trybit Logic obteve uma mediana maior em ambas questões. O jogo apresenta uma música polifônica durante todo seu tempo de jogo, além de conter sons de efeito para indicar ações no jogo, o que pode ter causado desconforto em alguns usuários, razão pela qual sua mediana nesta questão se apresenta mais alta. Já em Andor, o jogo somente apresenta sons em determinadas ações do jogo, sem música de fundo. Conforme item 2 da Fig. 7, nota-se que o ícone para que o som do jogo seja 
habilitado ou desabilitado está sempre presente na tela. Quanto a isso, um usuário relatou um bug, nos qual após desabilitar o som, ele seguiu funcionando.

Em Trybit Logic, é necessário navegar até configurações para desabilita-lo, conforme Fig. 8, não sendo difícil de encontrar, somente exigindo uma maior navegação entre telas.

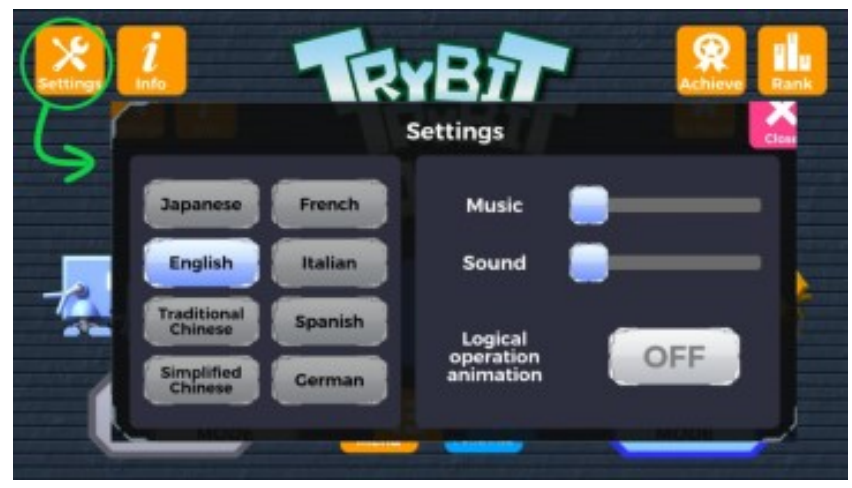

Figure 8: Jogo Trybit: desabilitar som

Observando a Tab. 5, nota-se que a questão A.4, do jogo Andor, apresenta uma baixa mediana devido às reclamações dos usuários em relação ao jogo apresentar o pop-up com as explicações em cada tentativa de navegar entre os níveis, conforme Fig. 9.

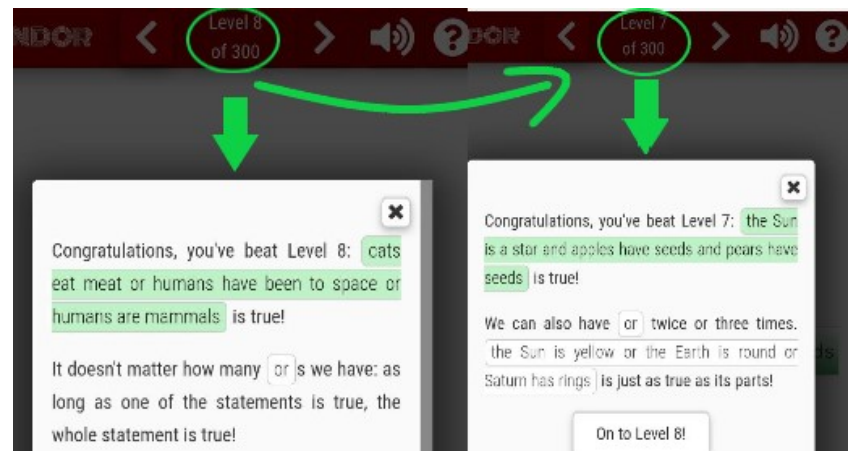

Figure 9: Jogo Andor: navegar entre os níveis

Das questões apresentadas na Tab. 5 referentes ao jogo Trybit Logic, percebe-se que as questões A.1, A.6 e A.7 apresentam uma mediana alta, pois o Puzzle Mode é o primeiro a ser exibido ao iniciar a aplicação e, conforme Fig. 10, as funcionalidades para reiniciar o nível após finalizá-lo (item 1) ou retomar ele do princípio (item 2) do jogo são intuitivas.

Ainda sobre o jogo Trybit Logic, na questão de código A.3 a mediana está baixa, pois os usuários avaliaram que as animações não interferiram na concentração durante o jogo. Já na questão A.9, a mediana não foi a maior pois somente é possível voltar ao início do Defense Mode retornando até o menu inicial e após finalizar o mesmo. Além disso, por parte dos usuários, houve reclamações de que neste tipo de modo de jogo os painéis de bits percorrem a tela muito rápido, o que, para um iniciante no jogo, se torna complicado acompanhar.

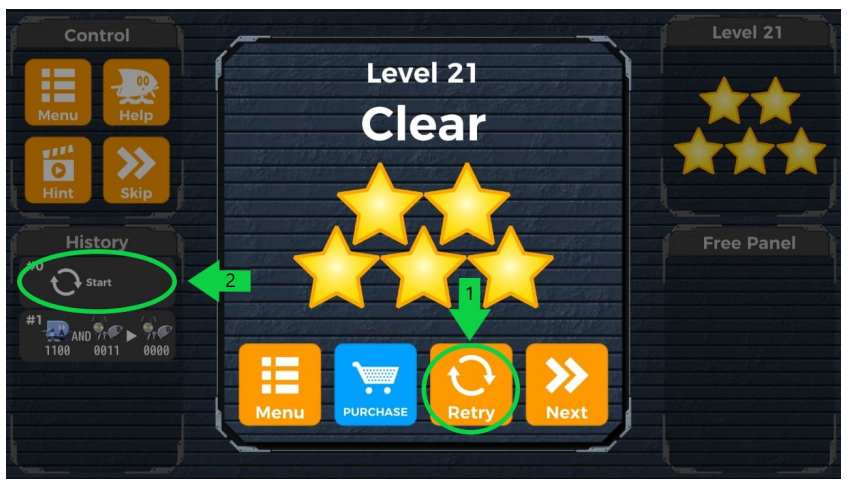

Figure 10: Jogo Andor: navegar entre os níveis

Em relação a mediana obtida na seção de questões B para o jogo Andor esperava-se um valor maior, pois o jogo é restrito e não apresenta muitas opções sobre como seguir, sendo um jogo simples e com um tutorial necessário somente para o entendimento dos operadores lógicos encontrados. Já o jogo Trybit Logic não funciona da mesma forma, é necessário que o tutorial seja visto e entendido para aprender tanto sobre o funcionamento do jogo como o entendimento dos operadores lógicos. Além de ser um jogo que não apresenta indicações sobre os erros que são cometidos durante os níveis.

Da seção de questões $C$, ambos aplicativos optam por diferentes dinâmicas para ensinar sobre os operadores lógicos e são considerados úteis se somados ao ensino teórico. Na seção D, a baixa avaliação sobre a interface do jogo Andor pode ter ocorrido devido às explicações que aparecem constantemente dificultando a navegação, apesar de ser uma interface simples, na qual visualizase somente uma tela. Em Trybit Logic percebe-se que a mediana para a mesma seção é alta, pois o jogo segue um padrão de cores em todos seus níveis e seus botões são intuitivos, sem ocorrências inesperadas.

Na seção E, sobre o ensino em sala de aula, os jogos apresentados podem ser úteis como complemento ao aprendizado do conteúdo trabalhado. Acredita-se que a baixa mediana (se comparada a mediana das outras seções) deva ocorrer em razão dos problemas anteriormente comentados. Destaca-se que a sintaxe da lógica proposicional é trabalhada no jogo Andor, enquanto Trybit Logic trabalha a semântica da mesma lógica. O idioma dos jogos pode ser um empecilho para o entendimento, porém, com o conhecimento prévio do professor sobre os jogos unido com o ensino em sala de aula, acredita-se que os jogos auxiliem no ensino-aprendizado.

Ao fim do teste, quando os usuários poderiam dar sua opinião final sobre o jogo, houveram relatos sobre a má usabilidade do jogo Andor, pois ao abrir a aplicação ele inicia sem indícios do que deve ser feito primeiro, o que pode ser justificado pela falta de um tutorial sobre funcionamento do jogo. Entretanto, também existiram relatos positivos, nos quais somente foi apontado como negativo o fato de aparecerem pop-ups constantemente e de o jogo ser inteiramente em inglês, sendo de difícil compreensão para os não conhecedores da língua. Embora Trybit Logic também utilize o idioma inglês, ele foi relatado pelos usuários como divertido. 
Ambos os jogos apresentam adversidades que atrapalham a sua manipulação, mas acredita-se que não interferem no aprendizado, uma vez que os pontos de dificuldade relatados não estão diretamente ligados a leitura e ao raciocínio para resolução dos desafios que os jogos apresentam. Em Trybit Logic, o Defense Mode, apesar das reclamações, pode se tornar mais fácil com uma maior habilidade do Puzzle Mode, já que a assimilação dos operadores lógicos será melhor trabalhada. No jogo Andor, alguns usuários relataram ter sido difícil compreender o que deveria ser feito ao inicia-lo.

\section{CONSIDERAÇÕES FINAIS}

Os problemas enfrentados nos cursos de Computação levam muitos discentes a evadir dos cursos escolhidos ou permanecerem retidos naquelas disciplinas que se apresentam como desafiadoras. Também é possível apontar que, nos cursos dessa área, existe a dificuldade do aluno em compreender o tema abordado e a dificuldade do professor em levar o conteúdo de uma maneira compreensível ao discente. [10]

Em disciplinas que apresentam um viés matemático e teórico, como Lógica Matemática (LM), os alunos encontram dificuldades devido a sua temática. Porém, sabe-se que é necessário o entendimento dos conceitos para que a prática seja bem aplicada. Estratégias de ensino, bem como metodologias que despertem o interesse do aluno e tornem o aprendizado mais atrativo, estão cada vez mais sendo estudadas e utilizadas.

O presente trabalho teve como objetivo a realização de um teste de usabilidade com os jogos Andor e Trybit Logic, os quais podem auxiliar no ensino-aprendizagem de Lógica Matemática. O objetivo principal do teste foi avaliar itens como interface das aplicações, facilidade de uso, além da possibilidade de uso em sala de aula.

Após realização do teste de usabilidade com os usuários e do comparativo das informações coletadas através dos formulários, constatou-se que Trybit Logic é um jogo fácil de manipular, respeita padrões de cores e utiliza um objetivo avaliado como divertido através do conceito de destruição de um bug para proteção do robô BITROBO. O jogo também foi avaliado como útil no ensinoaprendizado do conteúdo abordado na disciplina de LM.

Cabe destacar que antes da realização do teste, foi informado aos usuários que não seria permitido uso das teclas do smartphone durante a realização do roteiro e solicitado que, após o download dos jogos, eles não fossem manipulados até o início do teste. Porém, como destacado na Seção 4, houve uma mediana alta para a questão que abordava a facilidade de sair do jogo, sendo que nenhum dos jogos apresenta tal possibilidade sem o uso das teclas do smartphone. Durante o teste, um dos usuários relatou ter utilizado os jogos antes da realização do mesmo.

Sabe-se que podem haver ameaças a validade do teste, pois mesmo notificando os usuários algumas restrições foram descumpridas, ele ocorreu de maneira remota, devido a situação atual de pandemia. Também foi notado que, pelos mesmos usuários utilizarem ambas aplicações, isso pode ter interferido na avaliação de cada um à cada jogo, mesmo que sejam distintos, porém se fosse viável, o teste teria ocorrido de maneira presencial em um ambiente controlado para tentar garantir uma maior preservação do cumprimento do que foi solicitado.

Portanto, o jogo Trybit Logic, por obter melhores resultados em relação a sua usabilidade e sendo o melhor apreciado entre os participantes se torna o recomendado àqueles interessados em uma maior fixação do conteúdo da disciplina de Lógica Matemática a partir de um jogo.

Como trabalhos futuros, pretende-se realizar testes de usabilidade com as demais aplicações encontradas e analisadas em [4] a fim de evidenciar os principais problemas encontrados quanto a usabilidade e utilidade. Assim, espera-se construir um protótipo de uma aplicação para dispositivos móveis com foco no ensinoaprendizado de LM, que tenha como objetivo auxiliar discentes e docentes em sala de aula.

\section{REFERENCES}

[1] SBC. Educação superior em computação Estatísticas - 2018. Sociedade Brasileira de Computação, 2018. Disponível em: https://www.sbc.org.br/documentos-dasbc/summary/133-estatisticas/1287-estatisticas-computacao-2018. Acesso em: 02 dez. 2020.

[2] Raphael Magalhães Hoed. Análise da evasão em cursos superiores: o caso da evasão em cursos superiores da área de computação. Master's thesis, Universidade de Brasília, 2016.

[3] João Stephan, Alessandreia Oliveira, and Marcelo Renhe. O uso de jogos para apoiar o ensino e aprendizagem de programação. In Anais do XXXI Simpósio Brasileiro de Informática na Educação, pages 381-390, Porto Alegre, RS, Brasil, 2020. SBC. doi: 10.5753/cbie.sbie.2020.381. URL https://sol.sbc.org.br/index.php/ sbie/article/view/12794.

[4] Bruna Dias and Alice Finger. Aplicativos para o ensino-aprendizagem de lógica matemática: qual a melhor escolha? In Anais do XXVI Workshop de Informática na Escola, pages 111-120, Porto Alegre, RS, Brasil, 2020. SBC. doi: 10.5753/cbie. wie.2020.111. URL https://sol.sbc.org.br/index.php/wie/article/view/12603.

[5] Adrian Fernandez, Emilio Insfran, and Silvia Abrahão. Usability evaluation methods for the web: A systematic mapping study. Information and Software Technology, 53(8):789-817, 2011. ISSN 0950-5849. doi: https://doi.org/ 10.1016/j.infsof.2011.02.007. URL https://www.sciencedirect.com/science/article/ pii/S0950584911000607. Advances in functional size measurement and effort estimation - Extended best papers.

[6] Natasha Valentim, Jacilane Rabelo, Williamson Silva, Wallison Coutinho, Álvaro Mota, and Tayana Conte. Avaliando a qualidade de um aplicativo web móvel através de um teste de usabilidade: um relato de experiência. In Anais do XIII Simpósio Brasileiro de Qualidade de Software, pages 255-262. SBC, 082014.

[7] Andreas Schäfer, Jan Holz, Thiemo Leonhardt, Ulrik Schroeder, Philipp Brauner, and Martina Ziefle. From boring to scoring - a collaborative serious game for learning and practicing mathematical logic for computer science education. Computer Science Education, 23(2):87-111, 2013. doi: 10.1080/08993408.2013. 778040.

[8] Nadia Parsazadeh, Rosmah Ali, Mehran Rezaei, and Sanaz Zolfaghar Tehrani. The construction and validation of a usability evaluation survey for mobile learning environments. Studies in Educational Evaluation, 58:97 - 111, 2018. ISSN 0191-491X. doi: https://doi.org/10.1016/j.stueduc.2018.06.002. URL http: //www.sciencedirect.com/science/article/pii/S0191491X17301943.

[9] Djone Kochanski e Shirlei Vendrami e Cesar Franca. Experimentos com jogo educacional em engenharia de software. Anais do Computer on the Beach, 0 (0):179-188, 2014. ISSN 2358-0852. doi: 10.14210/cotb.v0n0.pp.179-188. URL https://siaiap32.univali.br/seer/index.php/acotb/article/view/6395.

[10] Antonia Santos e Marizete Santos e Juliana Diniz. Mobilizando conhecimentos matemáticos através dos jogos digitais. Anais do Computer on the Beach, 0 (0):269-278, 2015. ISSN 2358-0852. doi: 10.14210/cotb.v0n0.p269-278. URL https://siaiap32.univali.br/seer/index.php/acotb/article/view/7042.

[11] R Liker. A technique for the measurement of attitudes. Archives of Psychology, 22:55, 1932. URL https://ci.nii.ac.jp/naid/10027917457/en/.

[12] Matthias Jenny. Andor: Learn Logic. Google Play Store, 2018. Disponível em: https://play.google.com/store/apps/details?id=io.cordova.andor. Acesso em: 17 julho. 2020.

[13] HIMACS, Ltd. Trybit Logic. Google Play Store, 2019. Disponível em: https://play.google.com/store/apps/details?id=jp.himacs.smartapp.trybitlogichl= pt_BRgl=US. Acesso em: 17 julho. 2020.

[14] Jakob Nielsen. Why you only need to test with 5 users. Nielsen Norman Group, 2000. Disponível em: https://www.nngroup.com/articles/why-you-only-need-to-test-with-5users/: :text=In\%20testing $\% 20$ multiple $\% 20$ groups $\% 20$ of,of $\% 20$ people $\% 20 \mathrm{in} \% 20$ each \%20group. Acesso em: 02 dez. 2020. 